\title{
RADIATING SPHERES IN GENERAL RELATIVITY WITHA MIXED TRANSPORT ENERGY FLOW
}

\author{
W. BARRETO \\ International Centre for Theoretical Physics, Trieste, Italy \\ and \\ Laboratorio de Física Teórica, Departamento de Física, Escuela de Ciencias, \\ Núcleo Sucre, Universidad de Oriente Cumaná, Venezuela \\ and \\ L. A. NŨ̃EZ* \\ International Centre for Theoretical Physics, Trieste, Italy \\ and \\ Laboratorio de Física Teórica, Departamento de Física, Facultad de Ciencias, \\ Universidad de Los Andes, Mérida, Venezuela
}

(Received 4 June, 1990)

\begin{abstract}
A semi-numerical method by Herrera et al. (1980) is extended to handle the evolution of general relativistic spheres where diffusion and free streaming radiation processes coexist. It is shown when mixed-mode radiation is present a very different hydrodynamic picture emerges from the models previously considered in both radiation limit. Characteristic times for free streaming, hydrodynamics, and diffusion processes are considered comparable. Hydrodynamics and radiation are strongly coupled and the particular equation of state of the model emerges as a very important element in the dynamic of the matter distribution.
\end{abstract}

\section{Introduction}

General relativity has proven to have very important influences on the hydrodynamics of the gravitational collapse leading to a supernova explosion (Takahara and Sato, 1984; Baron et al., 1985). In a supernova scenario virtually all the gravitational binding energy of the collapsing configuration is radiated away in a form of neutrinos. The coupling of this neutrino radiation to the material hydrodynamics is most likely crucial to deliver a supernova explosion (Burrows and Lattimer, 1986; Copperstein, 1988; Giovanoni et al., 1989).

The two possible limits for the escaping radiation are free streaming out and diffusion. In the free free-streaming out limit the radiation field is slightly coupled to the matter hydrodynamics; therefore, typical radiation times are much shorter than the corresponding hydrodynamic ones. But in the diffusion limit approximation, because the matter is highly opaque to the radiation, the opposite inequality is valid - i.e., radiation times are larger than hydrodynamic characteristic intervals.

Free streaming out is associated to the early epoch of the collapse, while the diffusion limit approximation is though to be valid at late stages. During the free-streaming

* Postal address: Apartado 54, Mérida 5101A, Venezuela.

Astrophysics and Space Science 178: 261-275, 1991.

(C) 1991 Kluwer Academic Publishers. Printed in Belgium. 
phase, at most, $1 \%$ of the total amount o radiated binding energy is released in $10^{-4}$ of the total characteristic time for a supernova processes (Cooperstein, 1988). Between these two limits radiation and hydrodynamics are strongly coupled and have important mutual influences.

Some years ago there have been reported a semi-numerical method to follow the collapse of a general relativistic radiating matter configuration in a free-streaming limit approximation (Herrera et al., 1980; Herrera and Núñez, 1990, for a review). This method, extended to follow the collapse in the diffusion limit approximation (Herrera et al., 1987a), has recently been used to study the influence of diffusion processes in radiating general relativistic spheres (Barreto et al., 1989; Herrera et al., 1990). From this two opposite limits there have emerged qualitatively different hydrodynamic pictures for a gravitational collapse of a matter configuration having the same equation of state. In fact, Herrera et al. (1980, hereafter abbreviated to HJR), had found in Tolman VI-like radiating sphere, in the free-streaming limit approximation, outer matter shells bounce earlier than the inner ones. Recent calculations (Barreto et al., 1989) for the same Tolman VI model, but in the diffusion limit approximation, yield the opposite picture for the bouncing, i.e., inner shells bounce earlier than the outer ones.

In the present work we extend the recent work of Herrera et al. (1990). Free-streaming out and diffusion processes coexist. It is assumed for a local co-moving observer that free-streaming processes are proportional to diffusive ones. Within the framework of the above-mentioned semi-numerical method both the initial data and the profile of radiation are given at the surface of the matter distribution. In particular, the form of this profile is a very relevant physical input because it sets the time-scale for the hydrodynamic evolution of the sphere. Consistent with this approach the 'proportionality function' between both processes is calculated from the radiation profile at the surface of the configuration.

This work is organized as follows. In Section 2 the conventions used, the Einstein field equations, and an outline of the method are sketched. Section 3 contains a description of the models worked out. The discussion of the results is presented in Section 4.

\section{The Field Equations and the Method}

\subsection{THE ENERGY MOMENTUM TENSOR, THE METRIC AND THE EINSTEIN EQUATIONS}

Let us consider a nonstatic distribution of matter which is spherically symmetric. In radiation coordinates (Bondi, 1964) the metric takes the form

$$
\mathrm{d} s^{2}=e^{2 \beta}\left((V / r) \mathrm{d} u^{2}+2 \mathrm{~d} u \mathrm{~d} r\right)-r^{2}\left(\mathrm{~d} \vartheta^{2}+\sin ^{2} \vartheta \mathrm{d} \varphi^{2}\right)
$$

where $\beta$ and $V$ are functions of $u$ and $r ; u=x^{0}$ is a time-like coordinate, $r=x^{2}$ is the null coordinate and $\vartheta=x^{2}$ and $\varphi=x^{3}$ are the usual angle coordinates. It is worth 
mentioning that the $u$-coordinate is the retarded time as seen by distant observer and, therefore, $u$-constant surfaces are null cones open to the future.

The hydrodynamic scenario, as viewed by a local Minkowskian observer co-moving with the fluid, consists of:

(i) An isotropic fluid of density $\hat{\rho}$ and pressure $\hat{P}$.

(ii) Isotropic radiation energy density $3 \mathscr{P}$.

(iii) Unpolarized radiation energy flux density $\hat{\varepsilon}$ travelling in the radial direction.

(iv) Radiation energy flux density $\hat{q}$ diffunding in the radial direction.

Therefore, for this co-moving observer the covariant energy-momentum tensor is

$$
\hat{T}_{\mu \nu}=\left(\begin{array}{cccc}
(\hat{\rho}+3 \mathscr{P}+\hat{\varepsilon}) & -\hat{\varepsilon}-\hat{q} & 0 & 0 \\
-\hat{\varepsilon}-\hat{q} & (\hat{P}+\mathscr{P}+\hat{\varepsilon}) & 0 & 0 \\
0 & 0 & (\hat{P}+\mathscr{P}) & 0 \\
0 & 0 & 0 & (\hat{P}+\mathscr{P})
\end{array}\right)
$$

This energy-momentum tensor describing this fluid can be written, for a non-co-moving observer, in radiation coordinates as (Herrera and Núñez, 1990, for details);

$$
T_{\mu v}=(\rho+P) \mathbf{U}_{\mu} \mathbf{U}_{v}-P g_{\mu v}+\hat{\varepsilon} \mathbf{K}_{\mu} \mathbf{K}_{v}+\mathbf{Q}_{\mu} \mathbf{U}_{v}+\mathbf{Q}_{v} \mathbf{U}_{\mu},
$$

where $P=\hat{P}+\mathscr{P}, \rho=\hat{\rho}+3 \mathscr{P}$.

The four-velocity is given by

$$
\mathbf{U}_{\mu}=\delta_{\mu}^{0} e^{\beta}\left(1-\omega^{2}\right)^{-1 / 2}\left(\frac{V}{r}\right)^{1 / 2}+\delta_{\mu}^{1} e^{\beta}\left(\frac{V}{r}\right)^{-1 / 2}\left(\frac{1-\omega}{1+\omega}\right)^{1 / 2},
$$

the null four-vector is

$$
\mathbf{K}_{\mu}=\delta_{\mu}^{0} e^{\beta}\left(\frac{V}{r}\right)^{1 / 2}\left(\frac{1-\omega}{1+\omega}\right)^{1 / 2}
$$

and the heat flux vector can be written as

$$
\mathbf{Q}_{\mu}=\hat{q} e^{\beta}\left\{\omega\left(1-\omega^{2}\right)^{-1 / 2}\left(\frac{V}{r}\right)^{1 / 2} \delta_{\mu}^{0}-\left(\frac{1-\omega}{1+\omega}\right)^{1 / 2}\left(\frac{V}{r}\right)^{-1 / 2} \delta_{\mu}^{1}\right\}
$$

Note that

$$
\mathbf{Q}^{\mu} \mathbf{U}_{\mu}=0
$$

is valid for the above expression (5). Also, note that we have kept each contribution to the radiation fields, free streaming and diffusing, as viewed by the local co-moving observer. 
Outside matter, Equation (1) should represent Vaydia's metric, i.e.,

$$
\beta=0, \quad V=r-2 m(u), \quad \mathscr{E}=\frac{-m_{0}(u)}{4 \pi r(r-2 m(u))},
$$

where $m$ is an integration function depending on $u$, which is the same as the 'mass aspect' defined by Bondi et al. (1962). In addition, this 'mass aspect' coincides with the Schwarzschild mass in the static limit. Inside the matter configuration $m(u)$ is generalized to $\tilde{m}$ by considering everywhere

$$
V=e^{2 \beta}(r-2 \tilde{m}(u, r)) .
$$

Now, the Einstein field equations take the form (Herrera et al., 1987a; Herrera and Núñez, 1990) of

$$
\begin{aligned}
& e^{-4 \beta}\left(1-\frac{2 \tilde{m}}{r}\right)^{-1} T_{00}=\frac{\rho+P \omega^{2}}{1-\omega^{2}}+\hat{\varepsilon}\left(\frac{1+\omega}{1-\omega}\right)+\frac{2 \hat{q} \omega}{\left(1-\omega^{2}\right)}= \\
& =\frac{1}{4 \pi r(r-2 \tilde{m})}\left(-\tilde{m}_{0} e^{-2 \beta}+\left(1-\frac{2 \tilde{m}}{r}\right) \tilde{m}_{1}\right) \\
& e^{-2 \beta} T_{01}=\frac{\rho-P \omega}{1+\omega}-\hat{q}\left(\frac{1-\omega}{1+\omega}\right)=\frac{\tilde{m}_{1}}{4 \pi r^{2}}, \\
& \left(1-\frac{2 \tilde{m}}{r}\right) T_{11}=\frac{1-\omega}{1+\omega}(\rho+P)-2 \hat{q}\left(\frac{1-\omega}{1+\omega}\right)=\frac{r-2 \tilde{m}}{2 \pi r^{2}} \beta_{1} \\
& -T_{2}^{2}=-T_{3}^{3}=P= \\
& =\frac{-\beta_{01} e^{-2 \beta}}{4 \pi}+\frac{1}{8 \pi}\left(1-\frac{2 \tilde{m}}{r}\right)\left(2 \beta_{11}+4 \beta_{1}^{2}-\frac{\beta_{1}}{r}\right)+ \\
& +\frac{3 \beta_{1}\left(1-2 \tilde{m}_{1}\right)-\tilde{m}_{11}}{8 \pi r}
\end{aligned}
$$

Note that these equations are slightly different from those displayed in Herrera et al. (1987a). Our $\hat{q}$ differs from their $Q$ by a factor - i.e.,

$$
\hat{q}=\frac{\left(1-\omega^{2}\right)^{1 / 2}}{(1-2 \tilde{m} / r)^{1 / 2}} Q .
$$

Observe that, given the metric coefficients $\tilde{m}$ and $\beta$ for a radiating sphere with free streaming and heat flow ( $\hat{\varepsilon} \neq 0$ and $\hat{q} \neq 0$ ), the system $(10 \mathrm{a}-\mathrm{d})$ is underdetermined to find the physical variables $\omega, \rho, P, \hat{q}$, and $\hat{\varepsilon}$. Therefore, to obtain them unequivocally, it is necessary to introduce additional information about the nature of the physical processes involved within the matter configuration. For the present work we shall 
assume that

$$
\hat{\varepsilon}=\mathscr{F} \hat{q},
$$

i.e., for a local co-moving observer free-streaming processes are proportional to diffusive ones. In general this 'proportionality function' $\mathscr{F}$ should be a function of time $u$ and position $r$ and should be given by the microphysics of the radiation transfer. In this paper we do not discuss the mechanisms inducing either free streaming or diffusion; rather, we are interested to study to what extend they influence the hydrodynamic when both are considered simultaneously. For simplicity and without depriving it with all its physical meaning, we shall assume $\mathscr{F}$ as a funciton of $u$. With this, rather strong, assumption this $\mathscr{F}$ can be calculated at the boundary surface, provided the corresponding profiles of radiation emerging from the different processes considered. We shall refer to this point later in this section.

Now, defining two auxiliary functions

$$
\tilde{\rho}=\frac{\rho+P \omega}{1+\omega}-\hat{q}\left(\frac{1-\omega}{1+\omega}\right)
$$

and

$$
\widetilde{P}=\frac{P-\rho \omega}{1+\omega}-\hat{q}\left(\frac{1-\omega}{1+\omega}\right),
$$

which are referred to as the effective density and the effective pressure, respectively. Integrating the field equations (10b) and (10c) it can be obtained

$$
\tilde{m}=\int_{0}^{r} 4 \pi r^{2} \tilde{\rho} \mathrm{d} r
$$

and

$$
\beta=\int_{a(u)}^{r} \frac{2 \pi r^{2}}{r-2 \tilde{m}}(\tilde{\rho}+\tilde{P}) \mathrm{d} r .
$$

Consequently, $\tilde{m}(u, r)$ and $\beta(u, r)$ are written as functions of $\tilde{\rho}$ and $\tilde{P}$ in the same way that they are expressed in terms of $\rho$ and $P$ for the static limit.

\subsection{JUNCTION CONDITIONS AND THE SURFACE EQUATIONS}

For the method outlined below to be consistent, it is necessary to match the interior solution to the Vaidya metric at the surface of the distribution. This matching can be carried out either by using Darmois-Lichnerowicz conditions (Bonnor and Vickers, 1981 ) or, by requiring the continuity of the functions $\beta$ and $\tilde{m}$ across the boundary of the distribution and demanding that

$$
-\beta_{0 a}+\left(1-\frac{2 \tilde{m}_{a}}{a}\right) \beta_{1 a}-\frac{\tilde{m}_{1 a}}{2 a}=0
$$


at this boundary surface (Herrera and Jiménez, 1981). From now on the suffix $a$ indicates the corresponding quantity is evaluated at the surface $r=a(u)$.

If we use that $\beta$ is continuous and $\beta=0$ for the Vaidya metric, we may expand it near the boundary surface $r=a(u)$ as

$$
\beta_{0 a}+\dot{a} \beta_{1 a}=0
$$

where $\dot{a}=\mathrm{d} a / \mathrm{d} u$. If we substitute this last expression back into (14) and using field equations (10b) and (10c), we obtain

$$
\dot{a}=\frac{\left(1-\frac{2 \tilde{m}_{a}}{a}\right)\left\{\frac{\omega_{a} \rho_{a}-P_{a}}{1-\omega_{a}}+\hat{q}_{a}\right\}}{\left(\rho_{a}+P_{a}-2 \hat{q}_{a}\right)} .
$$

In radiation coordinates, in general, the velocity of matter is given by Bondi (1964) as

$$
\frac{\mathrm{d} r}{\mathrm{~d} u}=\frac{V}{r} \frac{\omega}{1-\omega} .
$$

Therefore, it follows that

$$
\dot{a}=\left(1-\frac{2 \tilde{m}_{a}}{a}\right) \frac{\omega_{a}}{1-\omega_{a}} .
$$

If we compare (16) and (18) we may get

$$
P_{a}=\hat{q}_{a} .
$$

Thus the pressure at the boundary of a heat conducting sphere is discontinuous and does not vanish (Santos, 1985; Herrera et al., 1987a, b). Instead, as seen from a local co-moving observer in radiation coordinates, it coincides with the value of the heat flux at the surface of the sphere. The crucial point of the HJR-method is the system of ordinary differential equations for quantities evaluated at the surface: the surface equations. Two of these equations are the same for any spherically-symmetric model. The first one is Equation (18), where

$$
\tilde{m}_{a} \equiv m(u)
$$

is the total mass. If we scale the radius $a$, the total mass $m$, and the time-like coordinate $u$ by the initial total mass $m(u=0)=m(0)$,

$$
A=a / m(0), \quad M=m / m(0) \quad \text { and } \quad u / m(0) \Rightarrow u ;
$$

and define

$$
F=1-2 M / A \quad \text { and } \quad \Omega=\frac{1}{1-\omega_{a}},
$$


Equation (2.19) can be written as

$$
\dot{A}=F(\Omega-1) \text {. }
$$

The second surface equation relates the total mass-loss rate with the energy flux through the surface. This can be obtained by evaluating the field equation (10a) at both sides of the boundary surface; it can be shaped as

$$
\frac{\dot{F}}{F}=\frac{Q+E^{i n}+(1-F)(\Omega-1)}{A},
$$

where

$$
Q \equiv 4 \pi a^{2} \hat{q}_{a}(2 \Omega-1) \quad \text { and } \quad E^{i n} \equiv 4 \pi a^{2} \hat{\varepsilon}^{i n}(2 \Omega-1) .
$$

If independent profiles for $F Q$ and $F E^{\text {in }}$ are given, then $\mathscr{F}$ can be written as

$$
\mathscr{F}=\frac{E^{\text {in }}}{Q} .
$$

The third surface equation can be obtained from the field equations (20b), (10c), and (10d) evaluated at $r=a(u)$ or, equivalently, by recalling the conservation equations

$$
\left(T_{1 ; \mu}^{\mu}\right)_{a}=0
$$

i.e.,

$$
\begin{aligned}
& -\left(\frac{(\tilde{\rho}+\tilde{P})}{(1-2 \tilde{m} / r)}\right)_{0 a}+\left(\frac{\partial \tilde{P}}{\partial r}\right)_{a}+ \\
& +\left[\frac{(\tilde{\rho}+\tilde{P})}{(1-2 \tilde{m} / r)}\left(4 \pi \tilde{P}+\frac{\tilde{m}}{r^{2}}\right)\right]_{a}=\frac{2 \omega_{a}}{a} \tilde{\rho}_{a}+\frac{2 P_{a}}{a} .
\end{aligned}
$$

We stress the conspicuous role played by the effective variables $\tilde{\rho}$ and $\widetilde{P}$ in this relation, which is the generalization of the Tolman-Oppenheimer-Volkov equation for nonstatic radiative situation. After some straightforward manipulations and in case that the effective density is separable, i.e., $\tilde{\rho}=h(r) g(u)$, it can be written as

$$
\frac{\dot{F}}{F}+(1-F) \dot{\Omega}=\tilde{\mathscr{G}}(F, \Omega, A) m(0),
$$

where

$$
\begin{aligned}
\tilde{G}(F, \Omega, A)= & \frac{2 \Omega F(1-F)}{A \tilde{\rho}_{a}} P_{a}-\frac{\Omega^{2} F \tilde{R}(1-F)}{\tilde{\rho}_{a}}+(F-1)(\Omega-1) \times \\
& \times\left[4 \pi a \tilde{\rho}_{a} \frac{(3 \Omega-1)}{\Omega}-\frac{3+F}{2 a}+\frac{\Omega F}{\tilde{\rho}_{a}} \tilde{\rho}_{1 a}+K(a) F\right],
\end{aligned}
$$


with

$$
\tilde{R}(u) \equiv\left[\frac{\partial \tilde{P}}{\partial r}+\frac{(\tilde{\rho}+\tilde{P})}{(1-2 \tilde{m} / r)}\left(4 \pi \tilde{P}+\frac{\tilde{m}}{r^{2}}\right)\right]_{a}
$$

and

$$
K(a) \equiv \frac{\mathrm{d}}{\mathrm{d} a} \ln \left[\frac{1}{a} \int_{0}^{a} r^{2}\left(\frac{h(r)}{h(a)}\right) \mathrm{d} r\right]
$$

See Herrera et al. (1987a) and Herrera and Núñez (1990) for details.

\subsection{THE METHOD}

The above considerations suggest the following procedure to obtain models of radiating fluid spheres.

(1) Take a static interior solution of the Einstein equations for a fluid with spherical symmetry,

$$
\rho_{\text {static }}=\rho(r) ; \quad P_{\text {static }}=P(r) .
$$

(2) Assume that the $r$ dependence of $\tilde{P}$ and $\tilde{\rho}$ is the same as that of $P_{\text {static }}$ and $\rho_{\text {static }}$ but taking care with the boundary condition which, because Equations (12a) and (12b), it reads

$$
\tilde{P}_{a}=-\omega_{a} \tilde{\rho}_{a}
$$

(3) With the $r$ dependence of $\tilde{\rho}$ and $\tilde{P}$ and if we use (13a) and (13b) we get $\tilde{m}$ and $\beta$ up to three functions of $u$.

(4) For these unknown functions of $u$, we have a system of ordinary differential equations ((20), (21), (23)) for quantities evaluated at the surface: the surface equations. The first two equations (Equations (20) and (21)) are model independent, and the third one depends of the particular choice of the equation of state.

(5) One has three surface equations (Equations (20), (21), and (23)) corresponding to: the boundary radius $A$, the velocity of the boundary surface (related to $\Omega$ ), the function $\tilde{m}$ evaluated at $r=a(u)$ (related to $F$ ) and the 'total luminosity' $F E^{i n}+F Q$. Providing one of these functions the system of surface equations can be integrated for any particular set of initial data.

(6) By substituting the result of the integration in the expressions for $\tilde{m}$ and $\beta$, these metric functions become completely determined.

(7) We obtained the 'proportionality function' $\mathscr{F}$, the complete set of matter variables $\rho, P, \omega, \hat{q}$, and $\hat{\varepsilon}$ can be algebraically found for any part of the sphere by using the field equations $(10 \mathrm{a}-\mathrm{d})$. 


\section{The Modeling Performed}

Within this section we shall work out two models, previously studied in both radiation limits (Herrera et al., 1980, 1987a, 1990; Barreto et al., 1989). These models are Schwarzschild-like and Tolman VI-like solutions. Although they are very simple models, they are not deprived of some interesting physical meaning. In the static limit the Schwarzschild-like homogeneous solution represents an incompressible fluid of constant density. The equation of state of the static Tolman VI solution approaches the one of a highly relativistic Fermi gas and, therefore, with the corresponding adiabatic exponent of $\frac{4}{3}$.

The first model considered is the Schwarzschild-like homogeneous model. The effective density is assumed to be

$$
\tilde{\rho}=f(u)
$$

and the effective pressure can be easily computed

$$
\tilde{P}=\tilde{\rho} \frac{(3-2 \Omega)\left(W^{2}-r^{2}\right)^{1 / 2}-\left(W^{2}-a^{2}\right)^{1 / 2}}{3\left(W^{2}-a^{2}\right)^{1 / 2}-(3-2 \Omega)\left(W^{2}-r^{2}\right)^{1 / 2}},
$$

where

$$
W^{2}=\frac{3}{8 \pi f(u)} .
$$

The surface equations are written as

$$
\begin{aligned}
& \dot{A}=F(\Omega-1), \\
& \dot{F}=\frac{F Q+F E^{i n}}{A}+(1-F) \frac{\dot{A}}{A}
\end{aligned}
$$

and

$$
\dot{\Omega}=\frac{\Omega}{1-F}\left[Q \frac{4 \Omega^{2}}{3 A}-\frac{3(1-F)^{2}}{2 A \Omega}(2 \Omega-1)(\Omega-1)-\frac{\dot{F}}{F}\right] .
$$

This system has been integrated by use of

$$
A(0)=5, \quad F(0)=0.6 \text { and } \Omega(0)=1
$$

as a set of initial data. It is also considered that the total radiated mass is $m_{\text {rad }} \cong 0.04 m(0)$. For the present modeling it is possible to split the total radiated mass in

$$
m_{\mathrm{rad}}=m_{d-\mathrm{rad}}+m_{f-\mathrm{rad}}
$$

where $m_{d-\mathrm{rad}}$ and $m_{f-\mathrm{rad}}$ are the portion of the initial mass radiated by diffusion and free streaming out processes, respectively. For the Schwarzschild-like homogeneous 
model we have used

$$
m_{d-\mathrm{rad}} \cong 2.5 \times 10^{-4} m(0) \text { and } m_{f-\mathrm{rad}} \cong 4.0 \times 10^{-2} m(0) .
$$

The second model is the Tolman VI-like model expressed by the effective variables

$$
\tilde{\rho}=\frac{3 h(u)}{r^{2}}
$$

and

$$
\tilde{P}=\frac{\tilde{\rho}}{3}\left(\frac{1-9 k(u) r}{1-k(u) r}\right)
$$

The functions $h(u)$ and $k(u)$ are related through the boundary condition $\tilde{P}_{a}=-\omega_{a} \tilde{\rho}_{a}$ and, therefore, $k(u)$ can be expressed as

$$
k(u)=\frac{4 \Omega-3}{3 a(4 \Omega-1)} .
$$

For the present model the system of surface equations is

$$
\begin{aligned}
& \dot{A}=F(\Omega-1), \\
& \dot{F}=\frac{F Q+F E^{i n}}{A}+(1-F) \frac{\dot{A}}{A}
\end{aligned}
$$

and

$$
\dot{\Omega}=\frac{\Omega}{1-F}\left[4 Q F \Omega^{2}-\frac{F}{8 A}(1-F)(4 \Omega-3)(4 \Omega-1)-\frac{\dot{F}}{F}\right] .
$$

We integrate the above system using the initial data,

$$
A(0)=6.6667, \quad F(0)=0.7 \text { and } \Omega(0)=0.8571
$$

considering that

$$
m_{d-\mathrm{rad}}=2.0 \times 10^{-4} m(0) \text { and } m_{f-\mathrm{rad}}=2.1 \times 10^{-5} \mathrm{~m}(0) .
$$

As we have stressed above, the particular profiles for free streaming and diffusive processes are given independently (see Figures 1(a) and 1(b)) and the total luminosity contour is formed by the superposition of these two profiles. The significant values for their relative characteristic times and radiated energy are preserved. The integration is carried only during the stage of the collapse where both radiation processes are present and the running time is suggested by the behaviour of the matter variables themselves.

The Schwarzschild-like model is considered in Figures 2, 3, and 4. Figure 2 displays the profiles for the radial velocity; radiation energy flux density profiles are sketched in Figure 3 (heat flow) and Figure 4 (unpolarized radiation energy flux density). Figure 5 resume the radial velocity provides for a Tolman VI-like model. 


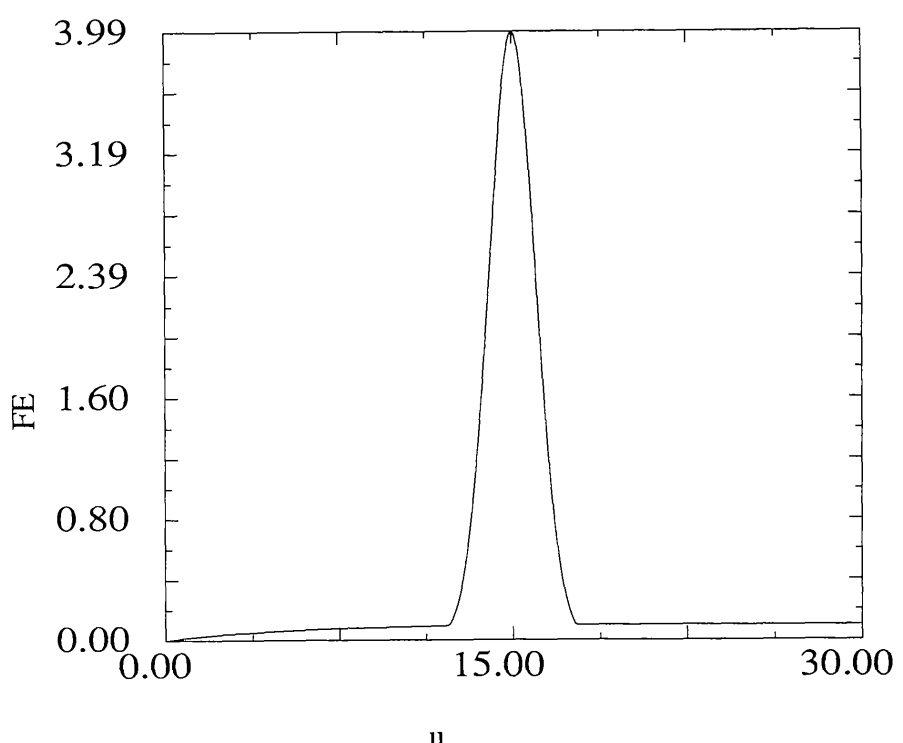

Fig. 1a. The pulse of radiation at the surface associated to free-streaming out processes. The $F E$ scale is multiplied by $10^{5}$.

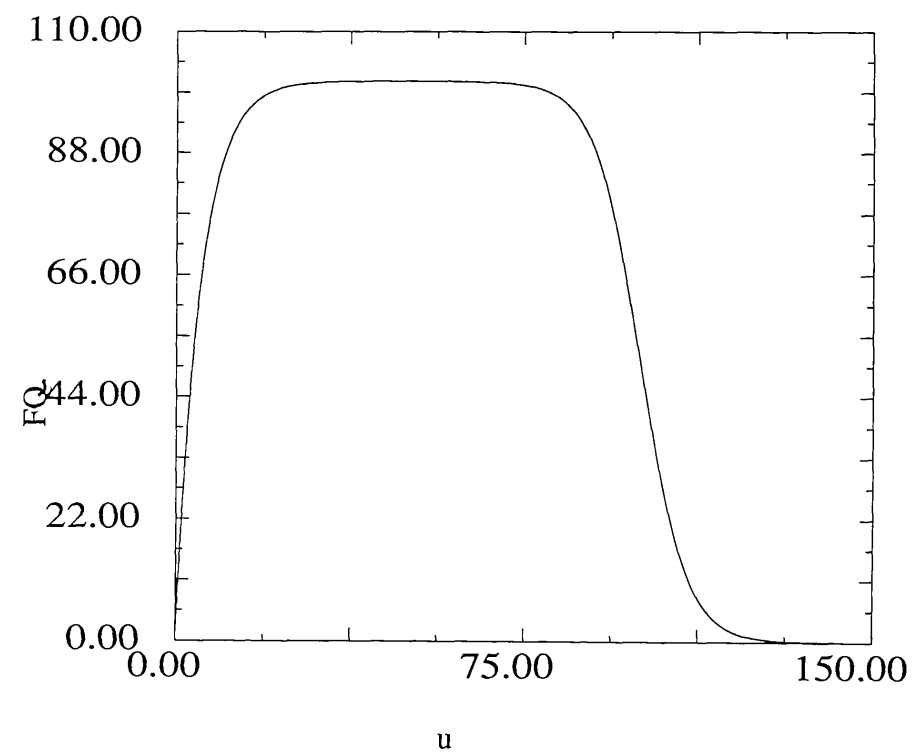

Fig. 1b. The radiation profile corresponding at the surface to diffusion processes. The $F Q$ scale has been multiplied by $10^{7}$ while the time-scale has been by $10^{-2}$.

\section{Conclusions}

We have seen, so far, that the HJR-method can be extended to include both diffusion and streaming out radiation. Although a model-dependent hydrodynamic picture seems to emerge from the present calculations, it is clear that the coexistence of both radiation fields induces a drastic changes in the dynamics of the previously studied models 
(Herrera et al., 1980, 1987a, 1990; Barreto et al., 1989). It is also possible to associate particular hydrodynamic behaviour to a specific radiation transfer scheme. In our simulations these models radiate much less energy but they have a qualitatively different evolution than those reported earlier (Herrera et al., 1980, 1987a, 1990; Barreto et al., 1989).

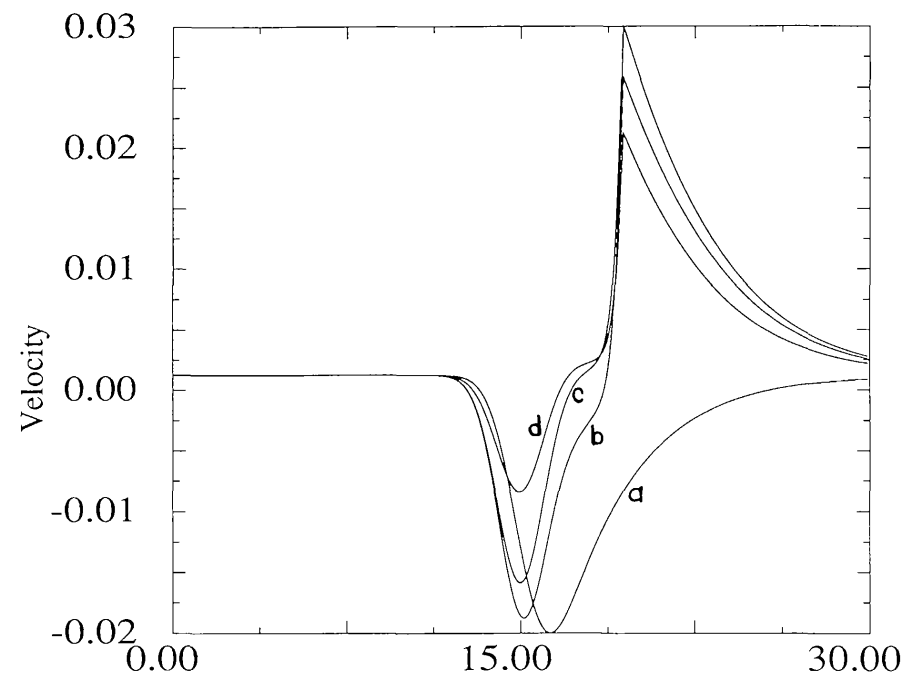

Fig. 2. The evolution of mass velocity for the Schwarzschild-like model. It is monitored at four different fixed mass shells. Curves labeled by (d), (c), (b), and (a) correspond to ratios $r / a_{0}=0.25,0.50,0.75,1.00$, respectively.

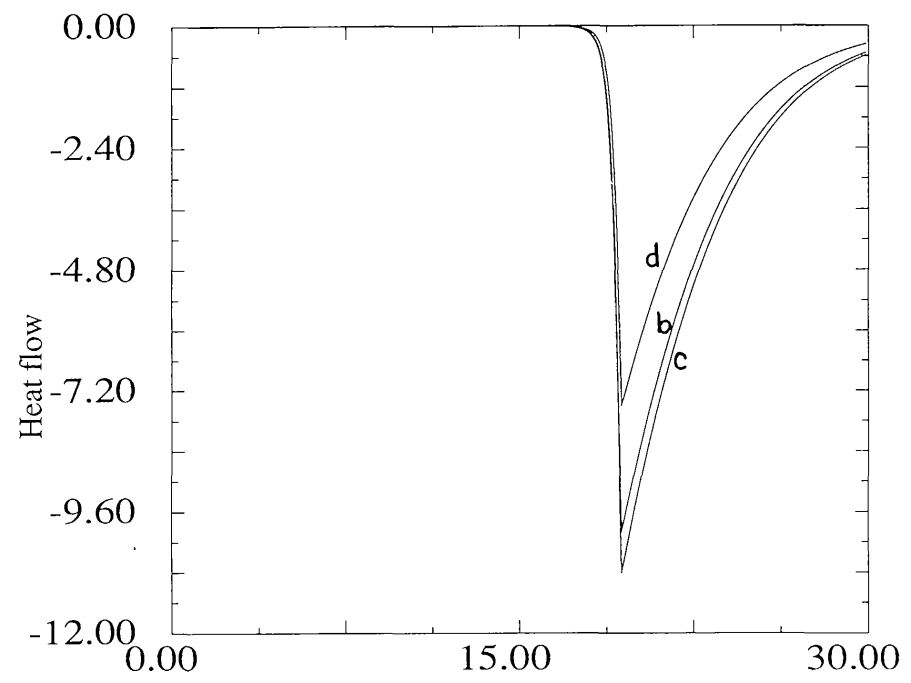

$\mathrm{u}$

Fig. 3. Heat flow profiles for the Schwarzschild-like model. Curves are labeled by (d), (c), and (b) correspond to ratios $r / a_{0}=0.25,0.50,0.75$, respectively. Heat flux values are multiplied by $10^{5}$. 
From Figure 2 it is clear that inner shells bounce for a Schwarzschild-like sphere. This bounce, absent in the streaming out limit (Herrera et al., 1980; Barreto et al., 1989), takes place when mainly diffusion processes are present (see Figures 3 and 4). Diffusion seems to be a very important mechanism for a Schwarzschild model because, although the contribution of these processes to the total radiated energy is fairly low, its hydrodynamic scenario resembles the one found for the diffusion limit approximation.

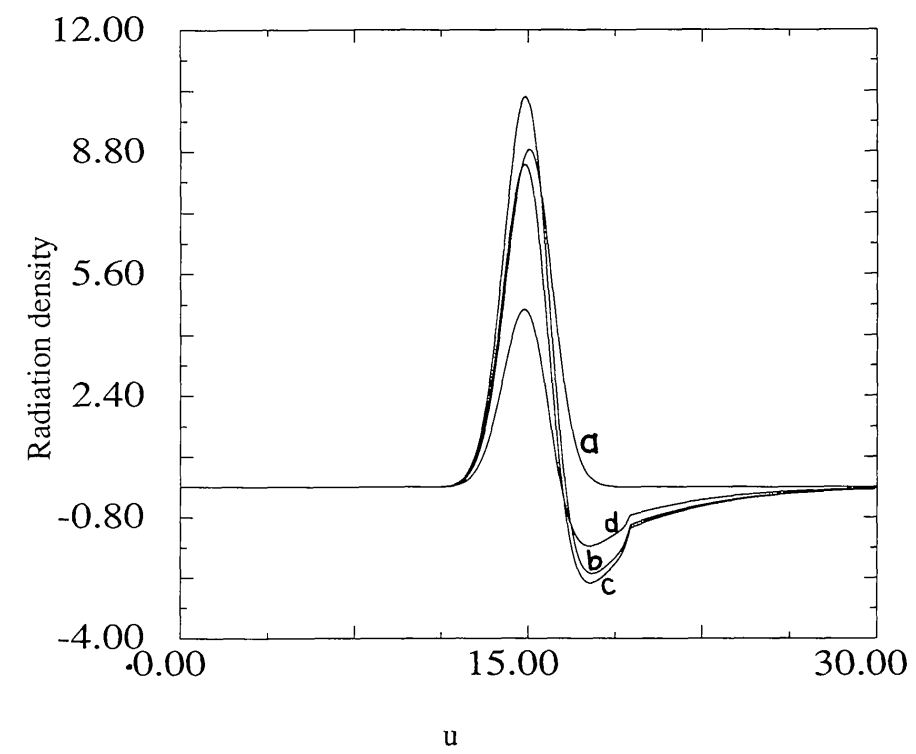

Fig. 4. Free-streaming out flux profiles for the Schwarzschild-like model. Curves are labeled by (d), (c), (b), and (a) correspond to ratios $r / a_{0}=0.25,0.50,0.75,1.00$, respectively. Radiation flux values are multiplied by $10^{5}$.

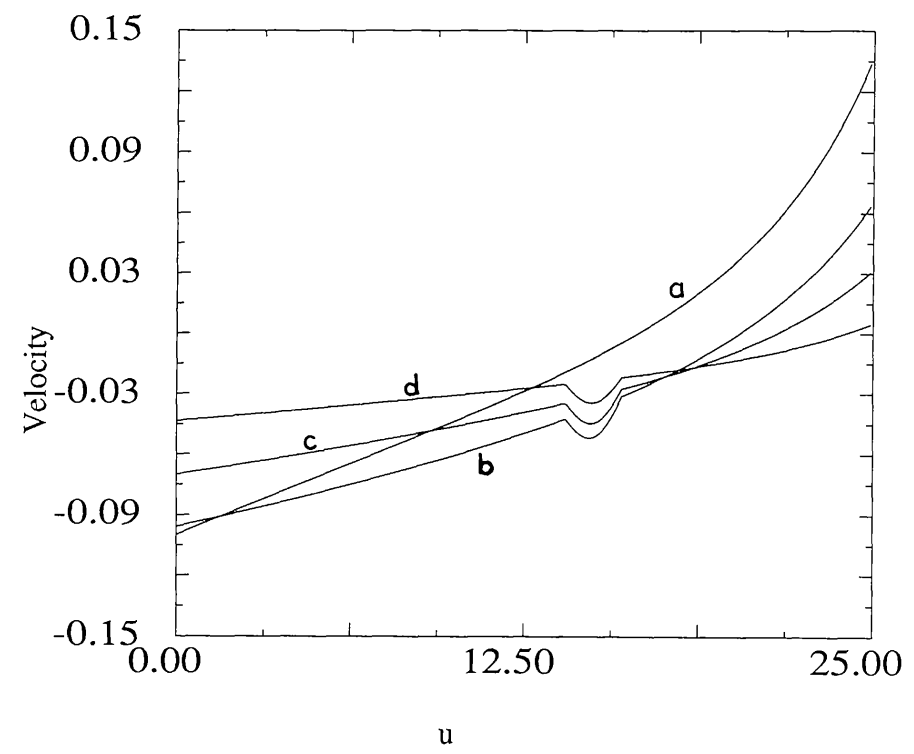

Fig. 5. The evolution of mass velocity for the Tolman VI-like model. Curves labeled by (d), (c), (b), and (a) correspond to monitoring shells at $r / a_{0}=0.25,0.50,0.75,1.00$, respectively. 
For the Tolman VI-like model an opposite picture is obtained (Figure 5). In this case the radiated mass emerging from free streaming is very low when it is compared with that emerging from the diffusion processes. Nevertheless, the hydrodynamics of this model is quite similar to the streaming out limit (Herrera et al., 1980; Barreto et al., 1989): inner regions bounce earlier than the outer ones. Therefore, we may conclude that, for the Tolman VI model, processes leading to streaming out are more relevant than those described by the diffusion.

These model-dependent conclusions could be understood in the following sense: when the characteristic times of free streaming, hydrodynamics, and diffusion proesses are considered comparable, hydrodynamics and radiation are strongly coupled; i.e., the particular equation of state chosen, emerges as a very important element in the dynamic of the matter distribution.

We would like to conclude with the following comments:

(a) All the values for the radiated mass either by streaming out and diffusion processes are the higher for a model to be physically reasonable.

(b) The obtained results are independent of the explicit dependence of the four-vector $\mathbf{Q}_{\mu}$ on the gradients of temperature.

(c) We have considered a very crude approximation for the radiation transfer within the matter distribution. The coexistence of free-streaming out and diffusion processes have been described by a 'proportionality function' $\mathscr{F}$. This function is assumed to be dependent only on $u$, in order to calculate it from the different radiation profiles at the surface of the configuration. Again, we harbour the hope that the astrophysical community, with much better information than we presently have, will provide the adequate microphysics description missing in our work.

\section{Acknowledgements}

The authors gratefully acknowledge the International Centre for Theoretical Physics (ICTP) for the financial and computer support. We would especially like to thank Professor Marek Abramowicz for the warm hospitality at ICTP Astrophysics Group. We have benefitted from the fruitful discussions with Leonardo Sigalotti. This work has been partially supported by the Consejo de Desarrollo Científico Humanístico y

Tecnológico de la Universidad de Los Andes, under project 379-89-05, and by the Consejo de Investigaciones de la Universidad de Oriente under project C.I. 5-022-00393-89.

\section{References}

Baron, E., Cooperstein, J., and Kahana, S.: 1985, Phys. Rev. Letters 12, 126.

Barreto, W., Herrera, L., and Santos, N. O.: 1989, Astrophys. J. 344, 158.

Bondi, H.: 1964, Proc. Roy. Soc. London 281, 39.

Bondi, H., Van der Burg, M. G. J., and Metzner, A. W. K.: 1962, Proc. Roy. Soc. London A281, 39.

Bonnor, W. B. and Vickers, P. A.: 1981, Gen. Rel. Grav. 13, 29.

Burrows, A. and Lattimer, J. M.: 1986, Astrophys. J. 307, 178. 
Cooperstein, J.: 1988, Phys. Reports 163, 95.

Giovanoni, P. M., Ellison, D. C., and Bruenn, S. W.: 1989, Astrophys. J. 342, 416.

Herrera, L. and Jiménez, J.: 1981, Phys. Rev. D28, 2987.

Herrera, L. and Núñez, L. A.: 1990, Fund. Cosmic Phys. (to appear).

Herrera, L., Jiménez, J., Barreto, W., Esculpi, M., LeDenmat, G., and Santos, N. O.: 1990, Astrophys. Space Sci. 165, 211.

Herrera, L., Jiménez, J., and Esculpi, M.: 1987a, Phys. Rev. D36, 2986.

Herrera, L., Jiménez, J., Esculpi, M. and Núñez, L.: 1987b, Phys. Letters A124, 248.

Herrera, L., Jiménez, J., and Ruggeri, G.: 1980, Phys. Rev. D22, 2305.

Santos, N. O.: 1985, Monthly Notices Roy. Astron. Soc. 216, 403.

Takahara, M. and Sato, K.: 1984, Prog. Theor. Phys. 72, 978. 